\title{
Prognostic markers in patients with ascites and hepatorenal syndrome
}

\author{
Leyla Nazal ${ }^{\mathrm{a}}$ and Andrés Cárdenas ${ }^{\mathrm{b}, *}$ \\ ${ }^{a}$ Gastroenterology Department, Air Force Hospital, Santiago, Chile \\ ${ }^{\mathrm{b}}$ GI/Endoscopy Unit, Institut de Malalties Digestives i Metaboliques, Hospital Clínic, University of Barcelona, \\ Barcelona, Spain
}

\section{Introduction}

Cirrhosis is a progressive liver disorder characterized by a distorted liver architecture due to fibrosis which eventually leads to portal hypertension. It is a common cause of mortality accounting for over 26,000 deaths annually in the United States [1]. The natural course of patients with cirrhosis is frequently complicated by the accumulation of fluid in the peritoneal space in the form of ascites. This is caused by an abnormal regulation of extracellular fluid volume which leads to alterations in renal function with renal sodium retention, solute-free water retention, and renal vasoconstriction. These changes are responsible for fluid accumulation in the form of ascites, dilutional hyponatremia and hepatorenal syndrome (HRS) respectively. Ascites is the most common complication of cirrhosis and poses and increased risk for infections, renal failure and mortality. Patients with cirrhosis and ascites have a poor prognosis and it is estimated that nearly half of these individuals will die in approximately 5 years without liver transplantation. Hypervolemic hyponatremia and HRS occur later and confer an even a worse prognosis. This article reviews common prognostic markers and models in cirrhotic patients with ascites, hypervolemic hyponatremia and HRS.

\footnotetext{
*Corresponding author: Andres Cardenas, MD, MMSc, GI Unit/ Institut de Malalties Digestives i Metaboliques, University of Barcelona, Hospital Clinic - Villarroel 170, Esc 3-2, 08036 Barcelona, Spain. Tel.: +34 93227 5513; Fax: +34 93227 9850; E-mail: acardena@clinic.ub.es.
}

\section{Ascites}

Ascites is defined as a pathological accumulation of free fluid in the peritoneal cavity. The development of ascites in a patient with cirrhosis defines a milestone as it is a condition associated with poor prognosis. Patients with compensated cirrhosis have a $30 \%$ risk of developing ascites at 5 years. Those that develop ascites have a probability of survival of $85 \%$ at 1 year and $56 \%$ at 5 years if they do not receive liver transplantation [2]. However, individual survival varies according to the degree of sodium retention, response to diuretics or associated complications (i.e. hemorrhage, infections or hepatocellular carcinoma). It is considered that patients with a first onset of ascites have better survival than those with previous episodes of ascites [3]. Additionally, patients with mild to moderate ascites (who have good response to treatment) have a better prognosis than patients with refractory ascites. The development of refractory ascites, characterized by an inability to resolve ascites with standard medical treatment, is associated with short term mortality and is a marker of poor prognosis with survival rate of about $50 \%$ at one year [4]. A number of factors associated with poor prognosis have been identified in patients with cirrhosis and ascites (Table 1). The most important factors in the prediction of poor prognosis are high Child-Pugh scores, increased serum creatinine, hyponatremia, intense sodium retention (urine sodium less than $10 \mathrm{mEq} /$ day), and low arterial pressure [5]. These factors are usually present in advanced liver dis- 
ease. Furthermore, it has been described that ascites related variables such as the ascitic fluid protein concentration and previous episodes of spontaneous bacterial peritonitis (SBP) add prognostic information to the Child Pugh Score [4,6]. A low total protein concentration in the ascitic fluid $(<15 \mathrm{gm} / \mathrm{L})$ is associated with an increased risk of SBP and in selected patients may indicate a need for antibiotic prophylaxis with oral quinolones to reduce the risk of SBP and HRS [6].

\section{Liver function}

A number of studies have shown that parameters of liver function correlate with prognosis and may be useful in clinical practice to estimate survival in the general population of patients with cirrhosis $[7,8]$. It is therefore not surprising that some liver function tests have a strong prognostic value in patients with cirrhosis and ascites. An increased serum bilirubin level or reduced serum albumin level is associated with a short survival in these patients $[9,10]$. By contrast, prothrombin activity has no predictive value in patients with ascites [5, $9,10]$. This lack of predictive value may be due to the fact that the prolongation of prothrombin time in patients with cirrhosis occurs very late in the evolution of the disease. In other disease states such as primary biliary cirrhosis or primary sclerosing cholangitis bilirubin levels in conjunction with albumin are considered very good markers of prognosis [11].

\section{Circulatory function}

The development of systemic hemodynamic disturbances in cirrhotic patients leads to effective hypovolemia, arterial hypotension, overactivity of vasoconstrictor systems including the renin-angiotensin system and the sympathetic nervous system and non-osmotic hypersecretion of arginine vasopressin (AVP). This circulatory dysfunction in patients with cirrhosis and ascites also correlates with survival and is a marker of poor prognosis [5]. Patients with low arterial pressure (mean arterial pressure $\leqslant 82 \mathrm{mmHg}$ ) have a poor prognosis compared to patients with normal arterial pressure [5,9]. Recent studies have shown that, in addition to the vascular disturbances, a relative inadequacy of cardiac output contributes to the renal hypoperfusion mainly in advanced HRS. Recent trials have shown that patients with ascites and a cardiac index below $1.51 / \mathrm{min} / \mathrm{m} 2$ had a poorer survival at 3,9 ,
Table 1

Adverse prognostic factors in cirrhosis with ascites

Exploratory findings:

Absence of hepatomegaly

Poor nutritional status

Previous ascites

Low arterial pressure

Esophageal varices

Liver tests

High serum bilirubin

Low serum albumin

Renal tests

Dilutional hyponatremia

Low urine sodium

Increased serum creatinine

Reduced water excretion after water load

Circulatory abnormalities

Low arterial blood pressure

High plasma renin activity

High plasma aldosterone

High plasma norepinephrine

\begin{abstract}
* Measurements of renal and hormonal function should be obtained after a minimum of 4 days on a low-sodium diet and without diuretics.
\end{abstract}

and 12 months than those with a cardiac index above $1.5 \mathrm{l} / \mathrm{min} / \mathrm{m} 2$ [12]. The activity of vasoconstrictor systems also has prognostic value in cirrhosis with ascites. Approximately $30 \%$ of patients with cirrhosis and ascites have normal levels of plasma renin activity and aldosterone. These patients have a better survival compared to patients with abnormal values of these parameters $[5,9,13]$. Patients with increased plasma renin activity and increased aldosterone and norepinephrine levels also have a high probability of developing HRS $[14,15]$.

\section{Renal function}

Renal dysfunction in cirrhosis is a consequence of circulatory disturbance, characterized by a low systemic vascular resistance and decreased effective arterial volume which leads to renal vasoconstriction and HRS $[14,16,17]$. The severities of renal and circulatory dysfunction are well established prognostic factors in patients with cirrhosis and ascites. In fact sodium retention, a highly prevalent renal function abnormality of cirrhosis, is associated with reduced survival [13]. Sodium excretion should ideally be measured in patients on a low-sodium diet of 70-90 mEq/day during 5-7 days and off diuretics. This parameter may indicate prognosis in patients with cirrhosis and ascites [5, 9]. Those who have a sodium excretion greater than 


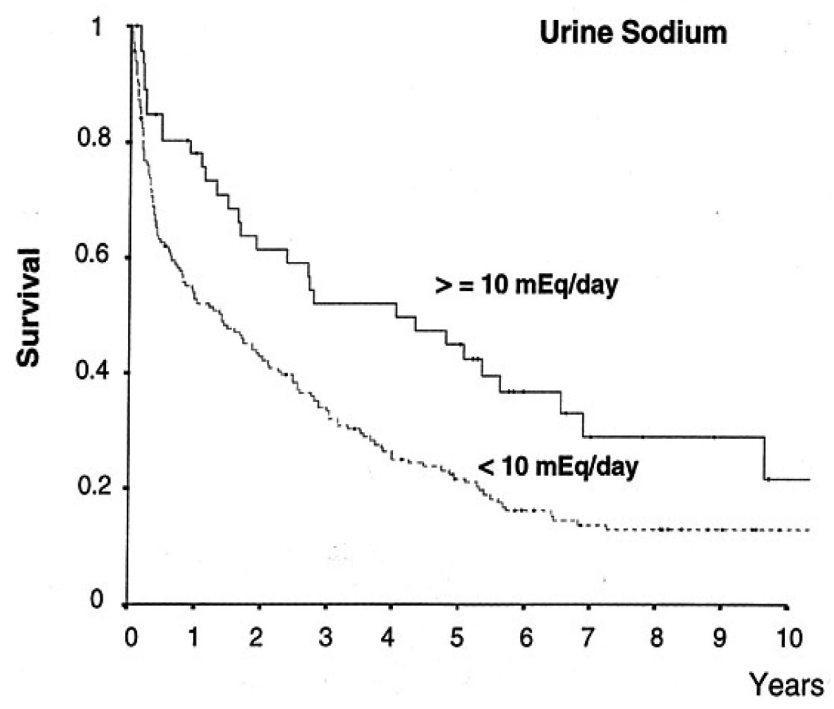

Fig. 1. Long-term survival according to sodium excretion in a series of patients with cirrhosis admitted to the hospital for the treatment of ascites.

sodium intake have a good prognosis, but patients with a markedly reduced sodium excretion $(<10 \mathrm{meq} / \mathrm{L})$ in relation with their intake have a poor outcome $[5,9,13$, 18] (Fig. 1).

An impaired ability to excrete solute-free water correlates with long-term prognosis in cirrhosis with ascites because it reflects the intensity of neurohumoral and circulatory dysfunction present in these patients [9, 19]. Patients with preserved renal ability to excrete free water have a better survival than patients with markedly impaired water excretion $[9,19]$. The predictive value of water excretion in the evaluation of long-term survival was confirmed in a large series of cirrhotic patients admitted to a single institution for the treatment of ascites [9]. Survival estimates for patients with normal diuresis ( $>8 \mathrm{~mL} / \mathrm{min})$ after a water load $(20 \mathrm{~mL} / \mathrm{kg}$ body weight of $5 \%$ dextrose IV) at 1, 5 and 10 years of follow-up were 85, 41 and $32 \%$. Corresponding values for patients with moderately reduced (3-8 $\mathrm{mL} / \mathrm{min})$ or severely-reduced $(<3 \mathrm{~mL} / \mathrm{min})$ diuresis after water load were only 55, 26 and $13 \%$, and 37,13 and $3 \%$, respectively. In this study, water excretion was the parameter with the strongest prognostic value compared to other parameters assessed.

Renal failure in cirrhosis is defined as an increase in serum creatinine $>1.5 \mathrm{mg} / \mathrm{dl}(20)$. Renal function as assessed with serum creatinine is an important marker of prognosis in patients with advanced cirrhosis. In fact the current allocation system of liver transplantation in the United States and other countries includes serum creatinine as a variable in the Model for End-Stage Liv- er Disease (MELD) scoring system. Renal function can be estimated by assessing glomerular filtration rate (GFR) either with the serum creatinine level, formulas that estimate GFR, or direct clearance methods with exogenous markers (21-23). However the most widely used parameter to estimate GFR in clinical practice is serum creatinine (21). Slight increases in serum creatinine (from 1.2 to $1.5 \mathrm{mg} / \mathrm{dl}$ ) are indicative of reductions in GFR and are associated with reduced survival. However, serum creatinine is highly influenced by factors such as decreased muscle mass and protein intake, so it can overestimate renal function in patients with cirrhosis [22,23]. Overestimation of renal function occurs more often in patients with a very low GFR.

The etiology of renal insufficiency in patients with cirrhosis also has a prognostic value in patients with cirrhosis [24]. The most common causes of renal failure in these patients are bacterial infections and volume depletion caused by bleeding or fluid losses. Drug induced renal failure (mainly from non-steroidal antiinflammatory drugs (NSAIDs) and intrinsic renal diseases (mainly glomerular disease associated with alcoholic liver disease, hepatitis $\mathrm{B}$ or $\mathrm{C}$ infection or other chronic kidney diseases) are less common causes. In a recent prospective study of 562 patients admitted to tertiary hospital for decompensated cirrhosis in a 6 year period [24], the most frequent cause of renal dysfunction was renal failure associated with infections, mainly $\operatorname{SBP}(46 \%)$, followed by hypovolemia-related renal failure (32\%), HRS (13\%), and parenchymal nephropathy $(9 \%)$. The 3-month probability of survival for all 
Table 2

Child pugh classification and model for end-stage liver disease model MELD

\begin{tabular}{lccc}
\multicolumn{4}{c}{ Child-Pugh Classification } \\
\hline & 1 & 2 & 3 \\
\hline Ascites & Absent & Mild & Moderate \\
Encephalopathy & Absent & $1-2$ & $3-4$ \\
Bilirubin mg/dL & $<2$ & $2-3$ & $>3$ \\
Bilirubin mg/dL & $<4$ & $4-10$ & $>10$ \\
Albumin gr/L & $>3,5$ & $2,8-3,5$ & $<2,8$ \\
INR & $<1,7$ & $1,8-2,3$ & $>2,3$ \\
\hline
\end{tabular}

Child A: $5-6$ points; Child B: $7-10$ points, Child C_ $10-15$ points

\begin{tabular}{ll}
\hline Score & Components \\
\hline MELD Score* & $9,2 *$ loge $($ creatinine mg/dL) $+3,8$ loge (bilirubin mg/dL) \\
& $+11,2$ loge $(\mathrm{INR})+6,4$ \\
MELD Sodium $^{* *}$ & MELD $+1,59^{*}(135-\mathrm{Na} \mathrm{mEq} / \mathrm{L})$ \\
\hline
\end{tabular}

*Values of creatinine, bilirrubin, INR lower than 1 are rounded to 1 . Serum creatinine values above $4 \mathrm{mg} / \mathrm{dL}$ are rounded to 4 . Patients on hemodyalisis are given a creatinine value of $4 \mathrm{mg} / \mathrm{dL}$. MELD scores ranged from 6 to 40 points.

** Values of serum sodium below $120 \mathrm{mEq} / \mathrm{L}$ are rounded to 120 . Values over

$135 \mathrm{mEq} / \mathrm{L}$ are rounded to 135 .

INR: international normalized ratio.

causes of renal insufficiency was $38 \%$ with a median survival of only 41 days. Patients with parenchymal nephropathy had the best survival (73\% probability of survival at 3 months), followed by patients with hypovolemia-related renal failure, who had a 3-month probability of survival of $46 \%$. Patients with renal failure associated with infections and those with HRS had the lowest 3-month probability of survival, which was 31 and $15 \%$, respectively.

\section{Hyponatremia}

Hyponatremia is common in advanced cirrhosis and is usually related to impaired solute-free water excretion primarily due to increased circulating levels of AVP which results in a disproportionate retention of water relative to sodium. Hyponatremia in cirrhosis may be due to hypovolemia or hypervolemia. In most cases it is due to a hypervolemic state due to a non-osmotic hypersecretion of AVP. In patients with cirrhosis and ascites the risk of developing hyponatremia is $15 \%$ at 1 year with a $25 \%$ probability of survival at 1 year [25]. Data from a prospective multicenter trial in nearly 1000 patients revealed that the prevalence of hyponatremia in cirrhosis as defined by a serum sodium level $\leqslant$ $135 \mathrm{mEq} / \mathrm{L}$ was $49 \%$; with levels $\leqslant 130 \mathrm{mEq} / \mathrm{L}, \leqslant 125$ $\mathrm{mEq} / \mathrm{L}$, and $\leqslant 120 \mathrm{mEq} / \mathrm{L}$ was $21.6 \%, 5.7 \%$, and $1.2 \%$, respectively [2]. In patients with refractory ascites or HRS, this proportion may increase up to 50\% [26]. Since hypervolemic hyponatremia is complication of cirrhosis that occurs in the late stages of the disease it is associated with the development of other complications of cirrhosis. Patients with hyponatremia have more severe liver disease, worse control of their ascites, a higher rate of hepatic encephalopathy, SBP and HRS when compared with patients without hyponatremia $(26,27)$ Regardless, both serum sodium and serum creatinine are independent prognostic factors of poor outcome in patients with cirrhosis. This is important since renal function (serum creatinine) is a variable included in the MELD scoring system for allocation of organs in liver transplantation. Hyponatremia also has clinical implications in patients undergoing LT. Patients that undergo liver transplantation with hyponatremia may be at risk for neurological complications, renal failure, and bacterial infections during the first month after transplantation and have increased 3-month mortality with respect to patients without hyponatremia [28,29].

\section{Prognostic models}

The two most used models to assess prognosis in decompensated cirrhosis are the Child-Pugh the MELD Score (Table 2) [30-32]. The main objective of prognostic models such as the Child-Pugh and MELD score is to provide precise information in order to make an accurate prediction of survival in a specific patient. As mentioned above, a number of variables with prognostic value, particularly those that that take into account renal and circulatory function have been identified in these patients. Nonetheless only one prognostic model that includes these variables (renal capacity to excrete 
a water load, mean arterial pressure, Child-Pugh class, and serum creatinine) has been proposed, however this test has not gained acceptance and may not be easily applicable in all centers [9]. For several decades, the Child-Pugh classification has been used in clinical practice to estimate survival of patients with ascites. This classification was originally designed to estimate the risk of death in cirrhotic patients submitted to surgical portosystemic shunts for the treatment of portal hypertension $[30,33,34]$. This system includes variables such as ascites, encephalopathy, serum bilirubin, serum albumin, and prothrombin time. Subsequent to its application to estimate surgical risk, the use of Child-Pugh classification was validated and extended to evaluate long-term prognosis of cirrhosis $[35,36]$. The simplicity of the Child-Pugh classification determined its wide use as prognostic model to evaluate survival in cirrhosis. However, the Child-Pugh classification has some drawbacks that limit its use as prognostic classification for patients with ascites. First, it does not include variables of renal or circulatory function, which are known to be very important prognostic factors in these patients. Second, prothrombin time which is one of the variables included in the classification has little prognostic value in patients with ascites $[5,10]$. Third, the score does not distinguish patients with serum bilirubin values of $10 \mathrm{mg} / \mathrm{dL}$ or $20 \mathrm{mg} / \mathrm{dL}$ or higher. Lastly, the Child-Pugh classification includes hepatic encephalopathy and ascites, two measures that are subject to a wide clinical interpretation and are much less objective. The main problem with the Child-Pugh classification is for patients that belong to the Child Pugh class B. It is well known that Child-Pugh class A patients usually show good midterm survival without transplantation unless other complications occur, while Child-Pugh class C patients are considered the conventional candidates for liver transplant. However, Child-Pugh class B patients are a heterogeneous group in which patients could remain stable for a long period or on the other hand can suddenly deteriorate into class C. Although these pitfalls were known for years, no other prognostic model of wide applicability and objective measures had been identified.

The MELD score was created in aims of better predicting survival in patients undergoing a transjugular intrahepatic shunt (TIPS) placement [32]. In this model, INR, total serum bilirubin level, serum creatinine level, and etiology of cirrhosis were used to predict survival following placement of a TIPS for any cause. This prognostic index was modified by removing the etiology and then implemented in the United States as the MELD Model to establish priority of patients awaiting liver transplantation [32]. The advantages of this system are that variables are objective and predictive. For instance, bilirubin is a robust variable also included in the Child-Pugh classification; renal dysfunction is a well-known variable associated with a poor prognosis in cirrhotic patients; and INR is the international normalized ratio for prothrombin time. The MELD model is also practical for in the risk stratification of patients undergoing TIPS, short term survival prediction of HRS and acute variceal bleeding [37-39] and risk stratification for non-transplant surgery [40, 41]. MELD has advantages over Child Pugh because it includes variables related to both liver and renal function. This score also excludes subjective variables, like encephalopathy and ascites. Nevertheless, studies indicate that some subsets of patients with cirrhosis may have high mortality despite low MELD scores [42]. Although patients with ascites with severe sodium retention and dilutional hyponatremia have a poor prognosis, they may have a low MELD score if they have normal creatinine levels. Since hyponatremia and impaired solute-free water excretion are events associated to development of HRS and have been associated with increased liver-related mortality [43] the addition of serum sodium to MELD score (MELD-Na) has been proposed as better prognostic model in patients awaiting liver transplantation [44]. In a study from the USA the ability of serum sodium to add prognostic capability to the MELD score was analyzed in adult primary liver transplant candidates with cirrhosis registered for transplantation during 2005 and 2006 [45]. Both the MELD score and the serum sodium concentration were predictors of mortality and when combined into a new MELD-Na score, those patients with low MELD scores benefited most from the new scoring system. Although the most accepted prognostic model in patients with cirrhosis awaiting LT in USA and several other countries is the MELD score, the Child-Pugh class still is considered an important prognostic factor specifically in those that are being considered for surgery or another major intervention.

\section{Hepatorenal syndrome}

HRS is a pre-renal renal failure without any identifiable kidney pathology that occurs in patients with advanced cirrhosis [20]. Due to the lack of specific diagnostic markers, the diagnosis of HRS is currently made using criteria to exclude other causes of renal failure 
Table 3

Diagnostic criteria of hepatorenal syndrome in cirrhosis*
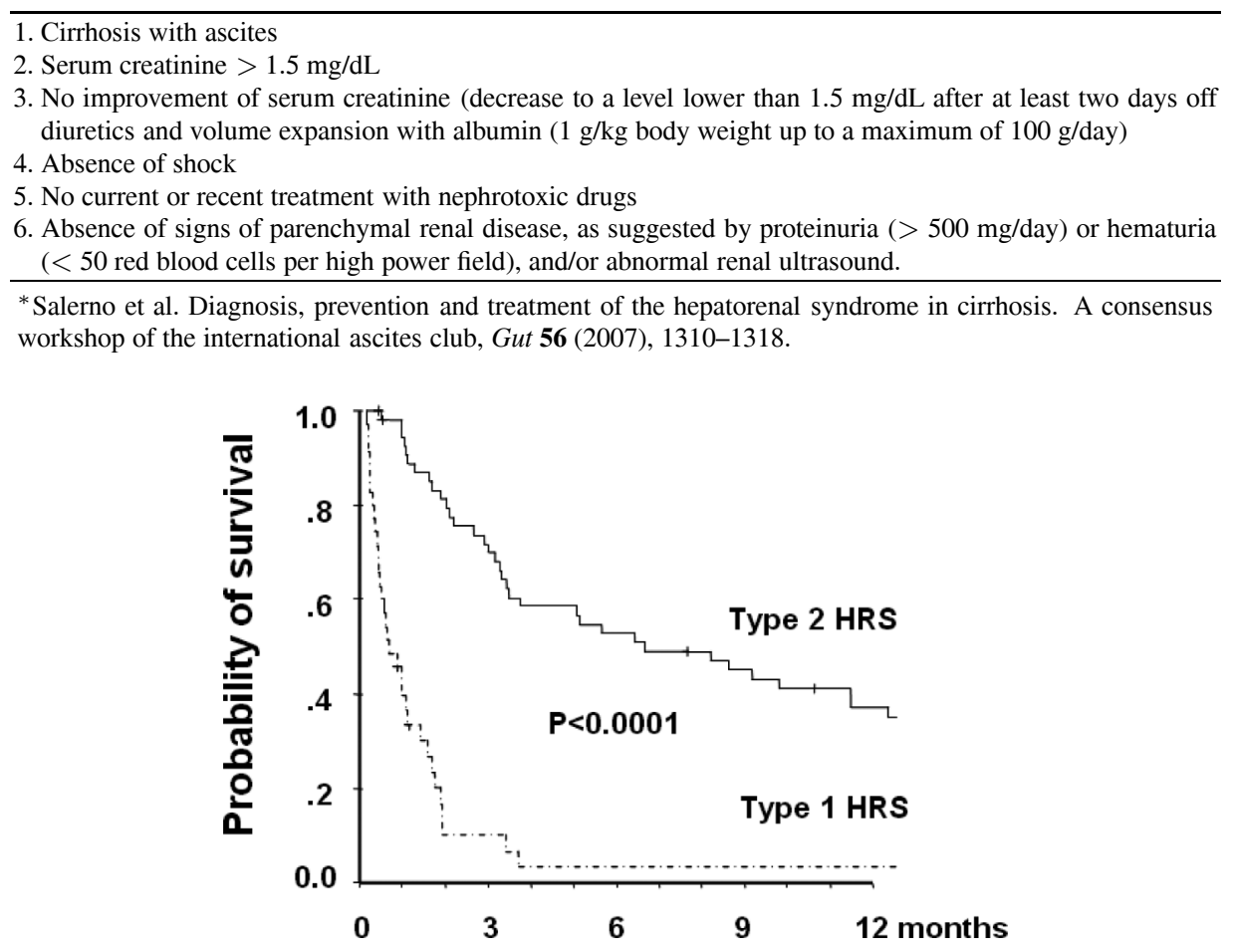

Fig. 2. Survival of patients with cirrhosis after the diagnosis of type 1 and type 2 hepatorenal syndrome.

that can occur in cirrhosis (Table 3). Patients who develop HRS have more advanced liver disease and features of circulatory dysfunction, with marked hypotension, low systemic vascular resistance, very high levels of renin activity, norepinephrine and AVP. These patients usually have low urine volume and intense sodium retention, with urine sodium $\leqslant 20 \mathrm{mEq} / \mathrm{L}$. The annual incidence of HRS in patients with ascites is approximately $8 \%$ and occurs in about $10 \%$ of hospitalized patients with cirrhosis and ascites. The probability of developing HRS in patients with cirrhosis and ascites is $18 \%$ at one year an $39 \%$ at five years [14]. There are two types of HRS; in Type 1 HRS renal function deteriorates rapidly with an increase in serum creatinine to a level higher than $2.5 \mathrm{mg} / \mathrm{dl}$ in less than 2 weeks. This type of HRS is associated with a very poor prognosis without treatment with a median survival time of only 2 weeks if untreated. In Type 2 HRS there is a steady impairment of renal function and serum creatinine levels usually range between $1.5-2.5 \mathrm{mg} / \mathrm{dl}$. Patients with Type 2 HRS have a median survival time of 6 months if not transplanted (Fig. 2). Patients with type 2 HRS may go on to develop type $1 \mathrm{HRS}$, either due to progression of disease or triggering factors such as bacterial infections.
Predictive factors associated with a greater risk of developing HRS have been described in cirrhotic patients with ascites without renal failure [14,15]. Patients with intense sodium retention ( $<10 \mathrm{mEq} /$ day $)$, spontaneous dilutional hyponatremia (serum sodium $<130 \mathrm{mEq} / \mathrm{L}$ ), a low mean arterial blood pressure $(<85 \mathrm{mmHg})$, decreased cardiac output $(<6.0 \mathrm{~L} / \mathrm{min})$, increased plasma renin activity, and increased aldosterone and norepinephrine levels have a high probability of developing HRS [14]. Recently it has been shown that cardiac dysfunction with reduction of cardiac index (CI) precedes the HRS $[12,15]$. In fact, CI is an independent predictor of development of HRS [15]. In a recent study, patients who died from type HRS 1 within 3 months had a lower CI than patients who survived this period. Patients with a cardiac output below $1.51 / \mathrm{min} / \mathrm{m}(2)$ had a significant poorer 12 month survival than those with a cardiac output above the mean level [52]. Other parameters, such as the degree of liver failure, as assessed by the levels of serum bilirubin, albumin, and prothrombin time, have not been consistently shown to predict the development of type $1 \mathrm{HRS}$.

The MELD score and the type of HRS (type 1) have an independent prognostic value for survival in both 
types of HRS [39]. The score can be useful in the management of patients with HRS, particularly for patients who are candidates for liver transplantation. Most patients with type 1 HRS have a MELD score $\geqslant 20$ [39]. A MELD score $>20$ in patients with HRS type 2 is associated with poor outcome compared to that of patients with MELD $<20$ so these patients should perhaps be given priority liver transplantation.

\section{Summary}

Patients with cirrhosis that develop ascites, hyponatremia and HRS have a poor prognosis. The prognostic factors of these complications are mainly related to the underlying circulatory dysfunction that occurs ion patienst with cirrhosis at an advanced stage. Other prognostic factors such as liver dysfunction are also important but do not seem to have a major influence on the outcome of these patients. The most common prognostic models in cirrhosis are the Child-Pugh score and the MELD score, both include variables that take into account liver and renal function. However the MELD score is the most commonly used prognostic model for organ allocation in liver transplant centers. More studies are needed in order to define if other variables of circulatory and renal dysfunction may improve the prognostic capability of these models.

\section{References}

[1] A.M. Minino, M.P. Heron and B.L. Smith, Deaths: Preliminary data for 2004, Natl Vital Stat Rep 54 (2006), 1-49.

[2] R. Planas, S. Montoliu, B. Balleste et al., Natural history of patients hospitalized for management of cirrhotic ascites, Clin Gastroenterol Hepatol 4 (2006), 1385-1394.

[3] P. Ginès, A. Cárdenas, V. Arroyo and J. Rodés, Management of cirrhosis and ascites, N Engl J Med 350 (2004), 1646-1654.

[4] R. Moreau, P. Delègue, F. Pessione et al., Clinical characteristics and outcome of patients with cirrhosis and refractory ascites, Liver Int 24 (2004), 457-464.

[5] J. Llach, P. Gines, V. Arroyo et al., Prognostic value of arterial pressure, endogenous vasoactive systems, and renal function in cirrhotic patients admitted to the hospital for the treatment of ascites, Gastroenterology 94 (1988), 482-487.

[6] P. Ginès and A. Cárdenas, The management of ascites and dilutional hyponatremia in cirrhosis, Semin Liver Dis 28 (2008), 43-58.

[7] P. Ginès, E. Quintero, V. Arroyo et al., Compensated cirrhosis: natural history and prognostic factors, Hepatology 7 (1987), 122-128.

[8] P. Schlichting, E. Christensen, P.K. Andersen, L. Fauerholdt, E. Juhl, H. Poulsen and N. Tygstrup, Prognostic factors in cirrhosis identified by cox's regression model, Hepatology 3 (1983), 889-895.
[9] G. Fernandez-Esparrach, A. Sanchez-Fueyo, P. Gines et al., A prognostic model for predicting survival in cirrhosis with ascites, J Hepatol 34 (2001), 46-52.

[10] F. Salerno, G. Borroni, P. Moser et al., Survival and prognostic factors of cirrhotic patients with ascites: A study of 134 outpatients, Am J Gastroenterol 88 (1993), 514-519.

[11] A. Pares and J. Rodes, Natural history of primary biliary cirrhosis, Clin Liver Dis 7 (2003), 779-794.

[12] A. Krag, F. Bendtsen, J.H. Henriksen and S. Moller, Low cardiac output predicts development of hepatorenal syndrome and survival in patients with cirrhosis and ascites, Gut 59 (2010), 105-110.

[13] V. Arroyo, J. Bosch, M. Mauri, F. Ribera, F. Navarro-Lopez and J. Rodes, Effect of angiotensin-ii blockade on systemic and hepatic haemodynamics and on the renin-angiotensinaldosterone system in cirrhosis with ascites, Eur J Clin Invest 11 (1981), 221-229.

[14] A. Gines, A. Escorsell, P. Gines et al., Incidence, predictive factors, and prognosis of the hepatorenal syndrome in cirrhosis with ascites, Gastroenterology 105 (1993), 229-236.

[15] L. Ruiz-del-Arbol, A. Monescillo, C. Arocena et al., Circulatory function and hepatorenal syndrome in cirrhosis, Hepatology 42 (2005), 439-447.

[16] S.A. Gonzalez and J.F. Trotter, Renal function and meld: Being direct is better, J Hepatol 52, 622-623.

[17] P. Gines and M. Guevara, Hyponatremia in cirrhosis: Pathogenesis, clinical significance, and management, Hepatology 48 (2008), 1002-1010.

[18] V. Arroyo, J. Bosch, J. Gaya-Beltran et al., Plasma renin activity and urinary sodium excretion as prognostic indicators in nonazotemic cirrhosis with ascites, Ann Intern Med 94 (1981), 198-201.

[19] P. Gines and M. Guevara, Hyponatremia in cirrhosis: Pathogenesis, clinical significance, and management, Hepatology 2008;48:1002-1010.

[20] F. Salerno, A. Gerbes, F. Wong et al., Diagnosis, prevention and treatment of the hepatorenal syndrome in cirrhosis. A consensus workshop of the international ascites club, Gut $\mathbf{5 6}$ (2007), 1310-1318.

[21] European Association for the Study of the Liver, P. Ginès et al., EASL clinical practice guidelines on the management of ascites, spontaneous bacterial peritonitis, and hepatorenal syndrome in cirrhosis, J Hepatol 53 (2010), 397-417.

[22] P. Ginès, A. Cárdenas and R.W. Schrier, Liver Disease and the Kidney, in: Diseases of the Kidney and Urinary Tract (8th ed.), R.W. Schrier ed., 2007, pp. 2179-2205.

[23] C. Francoz, D. Glotz, R. Moreau and F. Durand, The evaluation of renal function and disease in patients with cirrhosis, $J$ Hepatol 52 (2010), 605-613.

[24] M. Martin-Llahi, M. Guevara, A. Torre et al., Prognostic importance of the cause of renal failure in patients with cirrhosis, Gastroenterology, 2010 In press.

[25] P. Angeli, F. Wong, H. Watson and P. Gines, Hyponatremia in cirrhosis: Results of a patient population survey, Hepatology 44 (2006), 1535-1542.

[26] P. Gines, J. Uriz, B. Calahorra et al., Transjugular intrahepatic portosystemic shunting versus paracentesis plus albumin for refractory ascites in cirrhosis, Gastroenterology 123 (2002), 1839-1847.

[27] M. Guevara, M.E. Baccaro, J. Rios et al., Risk factors for hepatic encephalopathy in patients with cirrhosis and refractory ascites: Relevance of serum sodium concentration, Liver Int 30 (2010), 1137-1142. 
[28] M.C. Londono, M. Guevara, A. Rimola et al., Hyponatremia impairs early posttransplantation outcome in patients with cirrhosis undergoing liver transplantation, Gastroenterology 130 (2006), 1135-1143.

[29] A. Cardenas and P. Gines, Predicting mortality in cirrhosis serum sodium helps, N Engl J Med 359 (2008), 1060-1062.

[30] C.G. Child III and J.G. Turcotte, Surgery and portal hypertension. In: Child III CG Editor. The liver and portal hypertension, Philadelphia WB Saunders (1964), 50-56.

[31] F. Durand and D. Valla, Assessment of prognosis of cirrhosis, Semin Liver Dis 28 (2008), 110-122.

[32] P.S. Kamath, R.H. Wiesner, M. Malinchoc et al., A model to predict survival in patients with end-stage liver disease, Hepatology 33 (2001), 464-470.

[33] C.G. Child and J.G. Turcotte, Surgery and portal hypertension, Major Probl Clin Surg 1 (1964), 1-85.

[34] R.N. Pugh, I.M. Murray-Lyon, J.L. Dawson, M.C. Pietroni and R. Williams, Transection of the oesophagus for bleeding oesophageal varices, Br J Surg 60 (1973), 646-649.

[35] A. Propst, T. Propst, G. Zangerl, D. Ofner, G. Judmaier and W. Vogel, Prognosis and life expectancy in chronic liver disease, Dig Dis Sci 40 (1995), 1805-1815.

[36] E. Christensen, P. Schlichting, L. Fauerholdt et al., Prognostic value of child-turcotte criteria in medically treated cirrhosis, Hepatology 4 (1984), 430-435.

[37] L. Amitrano, M.A. Guardascione, R. Bennato, F. Manguso and A. Balzano, Meld score and hepatocellular carcinoma identify patients at different risk of short-term mortality among cirrhotics bleeding from esophageal varices, J Hepatol 42 (2005), $820-825$.
[38] N. Chalasani, C. Kahi, F. Francois et al., Model for end-stage liver disease (meld) for predicting mortality in patients with acute variceal bleeding, Hepatology 35 (2002), 1282-1284.

[39] C. Alessandria, O. Ozdogan, M. Guevara et al., Meld score and clinical type predict prognosis in hepatorenal syndrome: Relevance to liver transplantation, Hepatology 41 (2005), 12821289.

[40] S.H. Teh, J. Christein, J. Donohue et al., Hepatic resection of hepatocellular carcinoma in patients with cirrhosis: Model of end-stage liver disease (Meld) score predicts perioperative mortality, J Gastrointest Surg 9 (2005), 1207-1215; discussion 1215.

[41] S.H. Teh, D.M. Nagorney, S.R. Stevens et al., Risk factors for mortality after surgery in patients with cirrhosis, Gastroenterology 132 (2007), 1261-1269.

[42] E. Cholongitas, G.V. Papatheodoridis, M. Vangeli, N. Terreni, D. Patch and A.K. Burroughs, Systematic review: The model for end-stage liver disease - should it replace Child-Pugh's classification for assessing prognosis in cirrhosis? Aliment Pharmacol Ther 22 (2005), 1079-1089.

[43] S.W. Biggins, W.R. Kim, Terrault et al., Evidence-based incorporation of serum sodium concentration into meld, Gastroenterology 130 (2006), 1652-1660.

[44] M.C. Londono, A. Cardenas, M. Guevara et al., Meld score and serum sodium in the prediction of survival of patients with cirrhosis awaiting liver transplantation, Gut 56 (2007), 1283-1290.

[45] W.R. Kim, S.W. Biggins, W.K. Kremers et al., Hyponatremia and mortality among patients on the liver-transplant waiting list, N Engl J Med 4 (2008), 359, 1018-1026. 


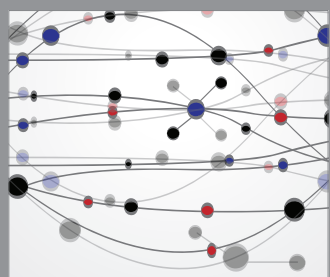

The Scientific World Journal
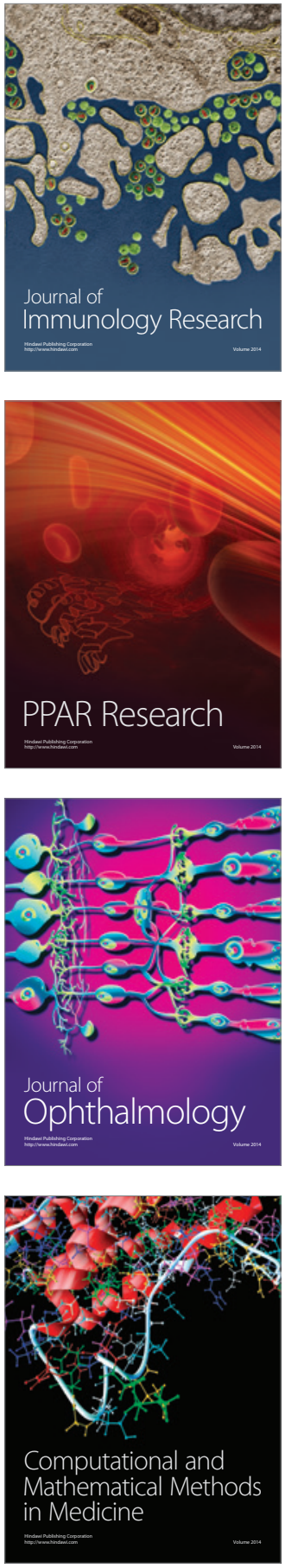

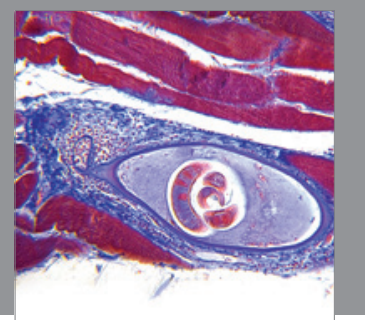

Gastroenterology

Research and Practice
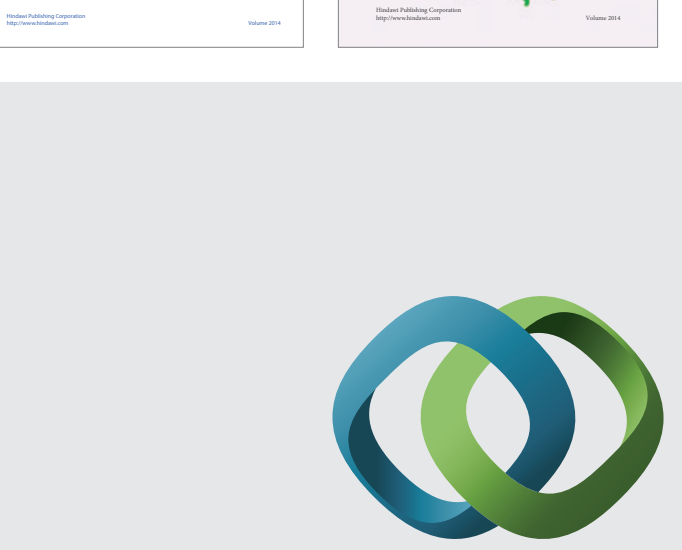

\section{Hindawi}

Submit your manuscripts at

http://www.hindawi.com
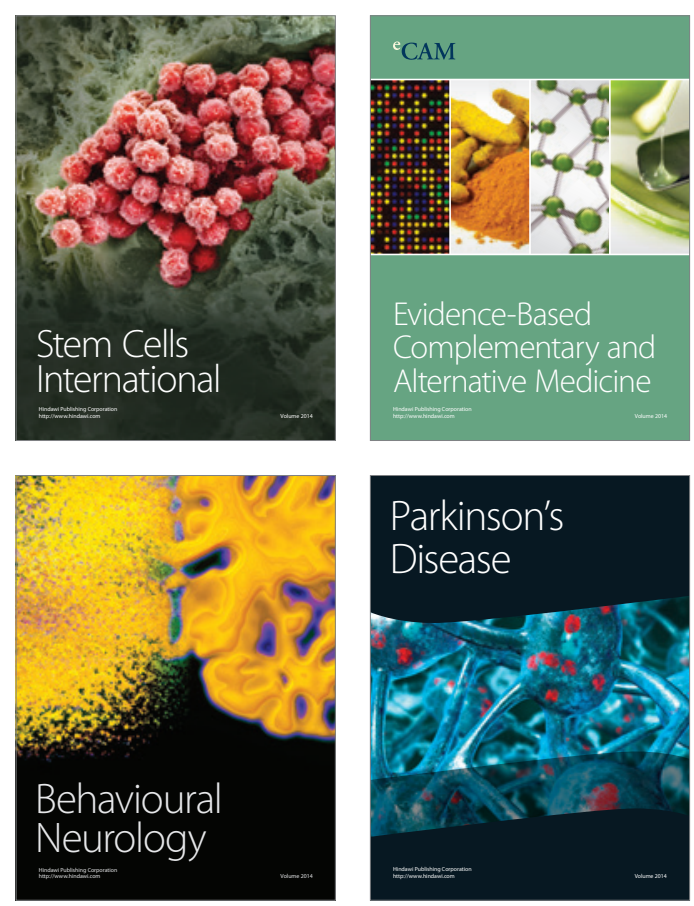

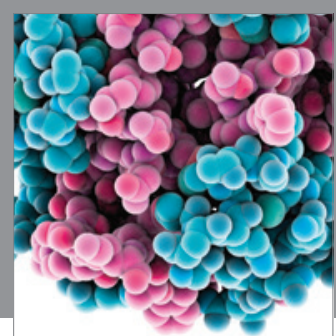

Journal of
Diabetes Research

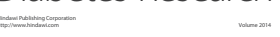

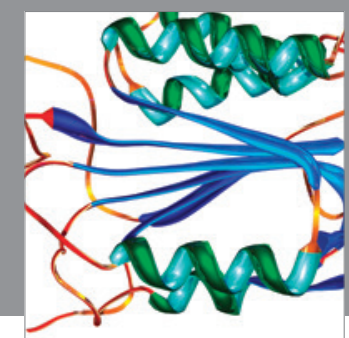

Disease Markers
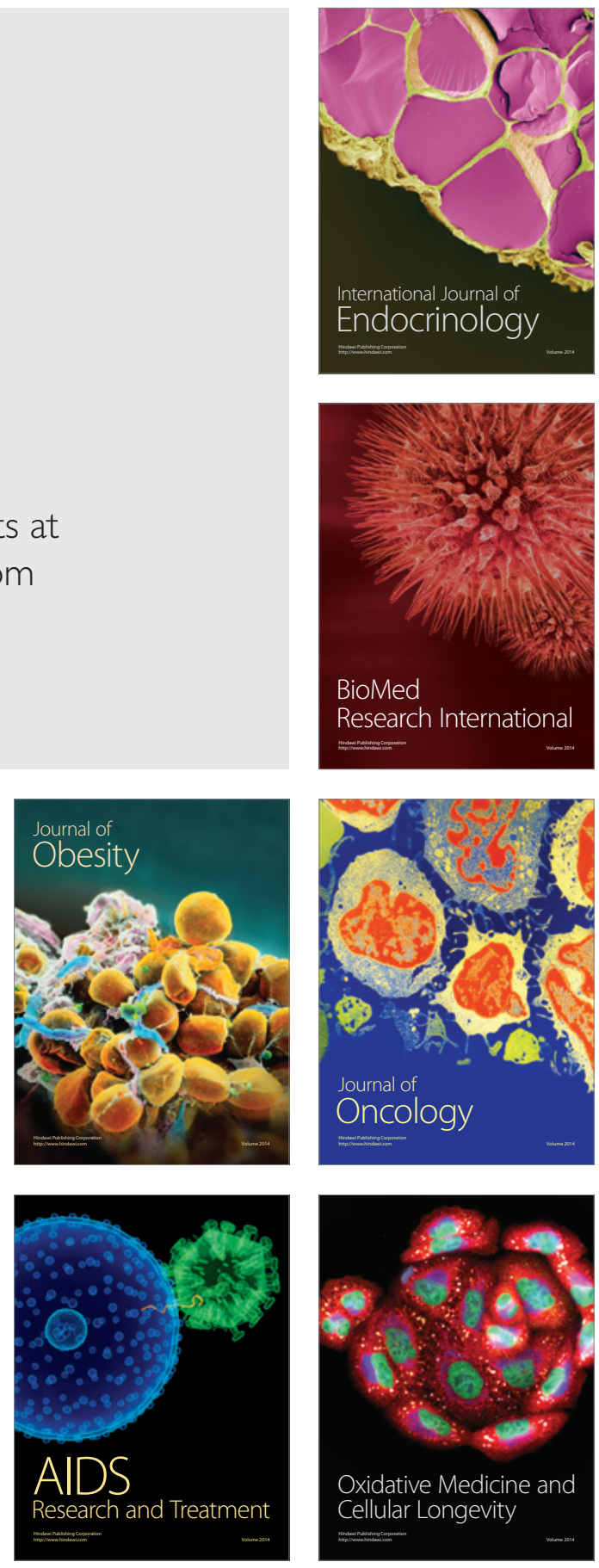